\title{
Assessing the Usefulness of Transition Metal Carbides for Hydrogenation Reactions
}

Received 00th August 2019, Accepted 00th January 20xx
Hector Prats, ${ }^{a}$ Juan José Piñero, ${ }^{a}$ Francesc Viñes, ${ }^{* a}$ Stefan T. Bromley, ${ }^{a, b}$ Ramón Sayós ${ }^{a}$ and Francesc Illas $^{\mathrm{a}}$
DOI: 10.1039/x0xx00000x

Transition Metal Carbides (TMCS) are proposed to replace scarce and expensive late Transition Metals (TMs) as heterogeneous catalysts, often implying hydrogenation reactions or steps. Present density functional theory based calculations support using group IV TMCs and $\delta$-MoC as viable alternative catalysts to TMs for so, given the moderate exoergicity and affordable reaction step energy barriers.

Transition Metal Carbides (TMCS) have been attracting much interest in the last decades for diverse technological applications in many fields, including heterogeneous catalysis, solid state chemistry, and materials science. ${ }^{1-5}$ A primary reason for this attention is the unique combination of physicochemical properties arising from the different degrees of ionic/covalent/metallic bonding exhibited in TMCs. In addition, some TMCs display catalytic activities similar or even better than those of noble and Pt-group metals ${ }^{6}$ for a considerable number of reactions including alkene hydrogenation, ${ }^{4,7}$ ammonia synthesis via the Haber-Bosch process, ${ }^{8}$ the water gas shift reaction, ${ }^{9}$ methanol synthesis from $\mathrm{CO}_{2}{ }^{10}$ fossil fuel refinement, ${ }^{11}$ and the production of other diverse chemicals compounds. ${ }^{12}$ Most of the aforementioned reactions have in common that they imply elementary hydrogenation steps which, to be carried out in an efficient fashion, require highly active and mobile (available) hydrogen atoms to be present on the TMC catalyst surface.

Hence, factors such as the types of hydrogen species and their stabilities play a central role in such hydrogenation reactions, ultimately determining the catalyst performance. Previous literature on the interaction of $\mathrm{H}_{2}$ on materials point for to the presence of diverse atomically chemisorbed $\mathrm{H}$ species, ${ }^{3,4}$ although other studies highlight the stability of molecularly physisorbed $\mathrm{H}_{2}{ }^{13}$ even surface Kubas $\mathrm{H}_{2}$ structures (i.e., chemisorbed $\mathrm{H}_{2}$ with an elongated bond), ${ }^{14,15}$ as well as $\mathrm{H}$ moieties present in the bulk, placed in defects or at interstitials. ${ }^{16}$ This blend of situations seems to back up a rich

\footnotetext{
${ }^{a}$ Departament de Ciència dels Materials i Química Física \& Institut de Química Teòrica i Computacional (IQTCUB), Universitat de Barcelona, c/ Martí i Franquès 111, 08028 Barcelona, Spain.

${ }^{b}$ Institució Catalana de Recerca i Estudis Avançats (ICREA), Passeig de Lluís Companys 23, 08010 Barcelona, Spain.

Electronic Supplementary Information (ESI) available: See DOI: $10.1039 / x 0 x x 00000 x$
}

adsorptive landscape of $\mathrm{H}_{2}$ on TMC surfaces, but, regardless of its central role in a plethora of catalyzed reactions, as above stated, the adsorption, desorption, dissociation, Kubas formation, and $\mathrm{H}$ and $\mathrm{H}_{2}$ mobilities on TMCs remain hitherto understudied, and so, poorly understood. Note that the present study focuses in heterogeneous catalysis applications, although the results can be of guidance for TMCs utilization as electrocatalysts for the Hydrogen Evolution Reaction $(H E R) .{ }^{17}$

Herein, we supply this necessary and lacking knowledge cornerstone by a thorough Density Functional Theory (DFT) based computational study of the interaction of atomic $(\mathrm{H})$ and molecular $\left(\mathrm{H}_{2}\right)$ hydrogen species on seven rocksalt TMCs. Their (001) surface has been considered, as previous studies have already demonstrated that this surface is the most stable. ${ }^{6}$ By considering a very wide range of molecular and atomic adsorption scenarios, we are able to establish the most energetically favorable $\mathrm{H}_{2}$ adsorption sites and dissociation pathways. The DFT based calculations have been carried out using the Perdew-Burke-Ernzerhof $(P B E)^{18}$ exchangecorrelation functional with the contribution of dispersion terms added through the D3 approach, as proposed by Grimme et al. (PBE-D3). ${ }^{19}$ The system total energy has been obtained by self consistently solving the Kohn-Sham equations with the valence electron density expanded in a plane wave basis set of $415 \mathrm{eV}$ of kinetic energy limit, and using periodic slab surface models. The Vienna Ab Initio Simulation Package (VASP) code has been used for all the optimizations. ${ }^{20}$ All other necessary computational details are provided in the Electronic Supplementary Information (ESI). This computational approach has been proven to be accurate when correlating simulations with experimental data on a number of de/hydrogenation reactions and/or interaction of $\mathrm{H}$ or $\mathrm{H}_{2}$ interactions with TMCbased catalysts. $4,5,21,25,27$

The $\mathrm{H}_{2}$ dissociation Transition States (TSs) have been located through the Climbing-Image Nudged Elastic Band ( $\mathrm{Cl}$ $N E B$ ) method, ${ }^{22}$ employing five intermediate images between reactants - initial state - and products - final state. These images were generated using the Atomic Simulation Environment $(A S E)^{23}$ and applying the Image Dependent Pair Potential (IDPP) procedure. $^{24}$ The gained TSs were characterized by frequency analysis ensuring they exhibit only one imaginary frequency. Note that favourable adsorption 
energies, $\Delta E_{a d s}$, are defined here negative, and, so, the more

energy values include the Zero Point Energy (ZPE) term, with negative the $\Delta E_{a d s}$, the stronger the bonding. All reported frequencies estimated within the harmonic approximation. Table 1 Calculated adsorption energy values $\left(\Delta E_{a d s}\right)$ for $\mathrm{H}_{2}, \mathrm{H}$, and $\mathrm{H}_{2, k u b a s}$ on the (001) surface of the studied TMCs. All values include the ZPE term.

\begin{tabular}{|c|c|c|c|c|c|c|c|}
\hline \multirow[b]{2}{*}{ Site } & \multicolumn{7}{|c|}{$\Delta E_{a d s}(\mathrm{eV})$} \\
\hline & $\mathrm{TiC}$ & $\mathrm{ZrC}$ & $\mathrm{HfC}$ & VC & $\mathrm{NbC}$ & $\mathrm{TaC}$ & $\delta$-MoC \\
\hline$H_{2}^{*(t o p-M)}$ & -0.10 & -0.11 & -0.11 & -0.05 & -0.06 & -0.13 & -0.42 \\
\hline$H_{2}^{*(t o p-C)}$ & -0.05 & -0.04 & -0.04 & -0.04 & -0.01 & -0.05 & -0.60 \\
\hline$H^{*}(t o p-M)$ & 1.51 & 1.33 & 1.20 & 0.60 & 0.35 & -0.06 & 0.21 \\
\hline$H^{*(t o p-C)}$ & -0.52 & -0.61 & -0.54 & 0.10 & 0.18 & 0.17 & -0.55 \\
\hline$H_{2 \text { Kubas }}^{*(t o p-C)}$ & -0.40 & -0.51 & -0.26 & 0.24 & 0.26 & 0.47 & -0.98 \\
\hline
\end{tabular}

Table 2 Calculated TS energy barriers for several steps involving adsorbed $\mathrm{H}_{2}$ and $\mathrm{H}$ species on the (001) surface of the studied TMCs. All values are given in eV and include the ZPE term.

\begin{tabular}{|c|c|c|c|c|c|c|c|}
\hline \multirow[b]{2}{*}{ Step } & \multicolumn{7}{|c|}{ Forward (reverse) energy barrier } \\
\hline & $\mathrm{TiC}$ & $\mathrm{ZrC}$ & $\mathrm{HfC}$ & VC & $\mathrm{NbC}$ & $\mathrm{TaC}$ & $\delta$-MoC \\
\hline$H_{2}^{*(t o p-M)} \stackrel{T S_{I}}{\leftrightarrow} H^{*(t o p-C)}+H^{*(t o p-M)}$ & $\begin{array}{c}0.68 \\
(0.00)\end{array}$ & $\begin{array}{c}0.52 \\
(0.06)\end{array}$ & $\begin{array}{c}0.48 \\
(0.06)\end{array}$ & $\begin{array}{c}0.65 \\
(0.00)\end{array}$ & $\begin{array}{c}0.60 \\
(0.04)\end{array}$ & $\begin{array}{c}0.47 \\
(0.11)\end{array}$ & $\begin{array}{c}0.39 \\
(0.31)\end{array}$ \\
\hline$H^{*(t o p-C)}+H^{*(t o p-M)} \stackrel{T S_{I I}}{\longleftrightarrow} 2 H^{*(t o p-C)}$ & $\begin{array}{c}0.10 \\
(1.72)\end{array}$ & $\begin{array}{c}0.03 \\
(1.60)\end{array}$ & $\begin{array}{c}0.12 \\
(1.50)\end{array}$ & $\begin{array}{c}0.14 \\
(0.53)\end{array}$ & $\begin{array}{c}0.38 \\
(0.52)\end{array}$ & - & $\begin{array}{c}0.19 \\
(0.95)\end{array}$ \\
\hline $2 H^{*(t o p-C)} \stackrel{T S_{I I I}}{\longleftrightarrow} H_{2, \text { Kubas }}^{*(\text { top }-C)}$ & $\begin{array}{c}1.11 \\
(0.47)\end{array}$ & $\begin{array}{c}1.42 \\
(0.71)\end{array}$ & $\begin{array}{c}1.44 \\
(0.63)\end{array}$ & $\begin{array}{c}0.66 \\
(0.62)\end{array}$ & $\begin{array}{c}0.82 \\
(0.92)\end{array}$ & - & $\begin{array}{l}1.07 \\
(0.96)\end{array}$ \\
\hline$H_{2, \text { Kubas }}^{*(\text { top }-C)} \stackrel{T S_{I V}}{\longleftrightarrow} H_{2}^{*(t o p-C)}$ & $\begin{array}{c}2.13 \\
(1.78)\end{array}$ & $\begin{array}{c}2.22 \\
(1.75)\end{array}$ & $\begin{array}{c}2.17 \\
(1.95)\end{array}$ & $\begin{array}{c}1.57 \\
(1.85)\end{array}$ & $\begin{array}{c}1.59 \\
(1.86)\end{array}$ & $\begin{array}{c}0.40 \\
(0.92)\end{array}$ & $\begin{array}{c}1.92 \\
(1.53)\end{array}$ \\
\hline$H^{*(t o p-C)}+H^{*(t o p-M)} \stackrel{T S_{V}}{\leftrightarrow} H_{2, \text { Kubas }}^{*(\text { top }-C)}$ & $\begin{array}{c}0.13 \\
(1.11)\end{array}$ & $\begin{array}{c}0.04 \\
(0.90)\end{array}$ & $\begin{array}{c}0.12 \\
(0.68)\end{array}$ & $\begin{array}{c}0.22 \\
(0.58)\end{array}$ & $\begin{array}{c}0.39 \\
(0.63)\end{array}$ & $\begin{array}{c}0.45 \\
(0.45)\end{array}$ & $\begin{array}{c}0.36 \\
(1.01)\end{array}$ \\
\hline$H_{2}^{*(t o p-M)} \stackrel{T S_{V I}}{\longleftrightarrow} H_{2}^{*(t o p-C)}$ & $\begin{array}{c}0.06 \\
(0.01)\end{array}$ & $\begin{array}{c}0.07 \\
(0.00)\end{array}$ & $\begin{array}{c}0.08 \\
(0.00)\end{array}$ & $\begin{array}{c}0.01 \\
(0.00)\end{array}$ & $\begin{array}{c}0.05 \\
(0.00)\end{array}$ & $\begin{array}{l}0.08 \\
(0.00)\end{array}$ & $\begin{array}{c}0.00 \\
(0.18)\end{array}$ \\
\hline$H_{2}^{*(t o p-M)} \stackrel{T S_{V I I}}{\longleftrightarrow} 2 H^{*(t o p-M)}$ & - & - & - & - & - & $\begin{array}{c}0.82 \\
(0.81)\end{array}$ & - \\
\hline $2 H^{*}(t o p-M) \stackrel{T S_{V I I I}}{\longleftrightarrow} H^{*}(t o p-C)+H^{*(t o p-M)}$ & - & - & - & - & - & $\begin{array}{c}0.77 \\
(0.42)\end{array}$ & - \\
\hline
\end{tabular}

The $\mathrm{H}_{2}$ molecular adsorption on each TMC (001) surface has been exhaustively sampled by considering three molecular conformations on the different high-symmetry, non-equivalent sites over the TMC surfaces. The results show that the $\mathrm{H}_{2}$ molecule ends up being physisorbed on top of either a surface metal atom (top-M) or a surface $\mathrm{C}$ atom (top-C), with top-M being the preferred site in almost all the studied TMCs, see Table 1, yet values range -0.01 to $-0.13 \mathrm{eV}$, thus, mostly due to dispersive forces between $\mathrm{H}_{2}$ and the TMC surfaces; see $\Delta E_{a d s}$ gained only at PBE level in Table S1 of the ESI. The $\delta$-MoC behaves different than the other TMCs, with a stronger adsorption of the $\mathrm{H}_{2}$ molecule on both top-M and top-C adsorption sites, the latter being the most stable one, with an $\Delta E_{a d s}$ of $-0.60 \mathrm{eV}$. The Kubas mode for adsorbed $\mathrm{H}_{2}$, previously described in the literature for a number of the here contemplated TMCs, ${ }^{14,25-27}$ corresponds to an almost dissociated $\mathrm{H}_{2}$ molecular state where both atomic $\mathrm{H}$ species are adsorbed on the same $\mathrm{C}$ atom, with $\mathrm{H}_{2}$ bond lengths ranging 1.68 to $1.80 \AA$, see Table S2 of the ESI, and having a non-negligible interaction between them, estimated to be in between -0.55 to $-0.92 \mathrm{eV}$, see Table S2 of the ESI. The adsorption energies reported on Table 1 show that the formation of the Kubas mode is actually thermodynamically favorable on group IV TMCs and $\delta$-MoC, with adsorption energies stronger than those of molecular $\mathrm{H}_{2}$ adsorption on top-C or top-M, yet Kubas $\mathrm{H}_{2}$ is not thermodynamically favored on group $\mathrm{V}$ TMCs. Interestingly, on $\mathrm{TaC}$ and $\delta$-MoC (001) surfaces the Kubas $\mathrm{H}_{2}$ leads to an elevation of the surface carbon atom, resulting in a final structure resembling a $\mathrm{CH}_{2}$ species adsorbed on a C-vacancy site, see Figure S1 of the ESI. However, the strong interaction of surface $C$ atoms with neighbouring surface metal atoms ${ }^{28}$ prevents considering the $\mathrm{CH}_{2}$ group as a carbene-type intermediate. Furthermore, although the formation of such $\mathrm{H}_{2}$ Kubas modes is typically assumed barrierless in the literature, ${ }^{14,25-27}$ we found that it actually involves a high energy barrier, as later discussed. Note that, in order to establish the existence of a Kubas formation barrier, one should first indeed find a stable physisorbed $\mathrm{H}_{2}$ state. However, finding this physisorbed site is elusive and very sensitive to the initial conditions of the geometry optimization; for instance, the $\mathrm{H}_{2}$ adsorbed on top-C requires an initial guess with the $\mathrm{H}_{2}$ molecule at least at $1.50 \AA$ above the $\mathrm{C}$ atom, and when initial conditions had the $\mathrm{H}_{2}$ closer to the surface, the adsorption directly evolves to the Kubas mode, giving the wrong impression that the process is non-activated. This could well be the reason why this barrier was not found in previous studies in the literature, yet it is crucial for providing a correct description of the ability of TMC surfaces to adsorb and dissociate $\mathrm{H}_{2}$, vide infra.

The atomic $\mathrm{H}$ adsorption was also studied considering six different non-equivalent sites on the TMC surfaces. Results show that, once again, top-M and top-C are the only stable 
adsorption sites for $\mathrm{H}$ adatoms, with top- $\mathrm{C}$ being the preferred site on all TMCs with $\Delta E_{a d s}$, when stable, ranging -0.52 to -0.61 $\mathrm{eV}$. The sole exception to this is $\mathrm{TaC}$, for which the top-M site is energetically preferred, see Table 1 , thus introducing itself as an appealing hydrogenating catalyst whenever the target molecule prefers to interact with a surface $\mathrm{C}$ atom. An interesting feature along group IV TMCs compared to group $\mathrm{V}$ is that the former leads to a much stronger adsorption of $\mathrm{H}$ on top-C compared to top-M sites, which in turn yields $\Delta E_{\text {ads }}$ differences between sites of up to $2.03 \mathrm{eV}$ on TiC while this

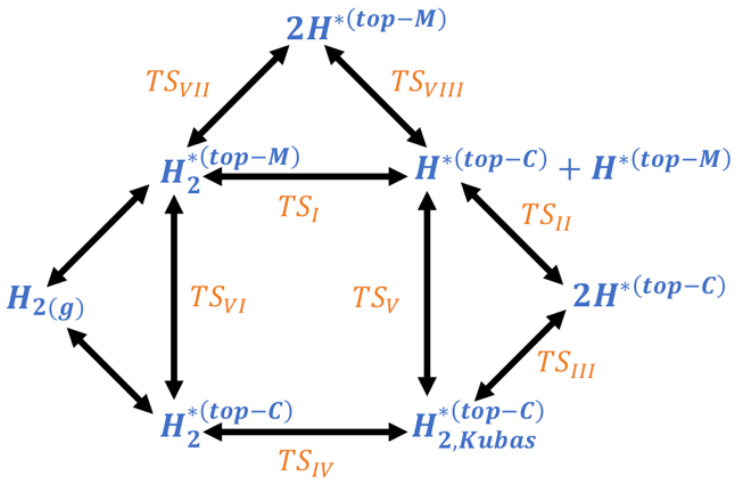

Figure 1 Graphical scheme showing the possible elementary steps connecting all adsorption minima and their corresponding transition states.

difference reduces to solely $0.17 \mathrm{eV}$ in $\mathrm{NbC}$. Results for atomic $\mathrm{H}$ adsorption also confirm a behaviour of $\delta$-MoC similar to with group IV with respect to $\Delta E_{\text {ads }}$ values on top-C and top-M sites, respectively, but with a reduced difference of $\Delta E_{\text {ads }}$ between sites of $0.76 \mathrm{eV}$. The effect of an adsorbed $\mathrm{H}$ on other adsorbed species is expected to be negligible, as both experimentally and theoretically proven on metallic surface systems. ${ }^{29,30}$ The optimized geometries for $\mathrm{H}_{2}$ and $\mathrm{H}$ adsorption on all the studied TMC surfaces are shown in Figure S1 of the ESI.

The dissociative $\mathrm{H}_{2}$ landscape over TMC (001) surfaces is more complex and richer than one could anticipate from chemical intuition. For instance, a $\mathrm{H}_{2}$ molecule landing on a TMC surface can physisorb on a top-M site, $H_{2}^{*(t o p-M)}$, and then dissociate into two vicinal chemisorbed $\mathrm{H}, H^{*(t o p-M)}$, species, or, alternatively, the $\mathrm{H}_{2}$ could physisorb on a top-C site, $H_{2}^{*(t o p-C)}$, and, subsequently, form a $\mathrm{H}_{2, \text { Kubas }}$ mode, $H_{2, \text { Kubas }}^{* \text { (top }-C)}$, to finally dissociate into two chemisorbed $\mathrm{H}$ atoms, $H^{*(t o p-C)}$. A graphical scheme including all the possible adsorption configurations and elementary steps connecting the diversity of adsorbing situations is shown in Figure 1. In total, eight elementary steps have been investigated, including $\mathrm{H}_{2}$ and $\mathrm{H}$ diffusion, and $\mathrm{H}_{2}$ dissociation. The corresponding $\mathrm{TS}_{\mathrm{I}-\mathrm{VIII}}$ values of these elementary steps are reported in Table 2 and the structures shown in Figure S2 of the ESI. Our results suggest that the formation of a $\mathrm{H}_{2, \text { Kubas }}$ from a physisorbed $\mathrm{H}_{2}$ molecule on top-C is very unlikely, due to the high predicted TS energy barriers, which range from 1.53 to $1.95 \mathrm{eV}$ in all TMCs except for $\mathrm{TaC}$, where the barrier is reduced to $0.92 \mathrm{eV}$, yet still fairly high. Instead, we found that the most feasible path for the $\mathrm{H}_{2, \text { Kubas }}$ formation starts from a physisorbed $\mathrm{H}_{2}$ molecule on a top-M site, followed by a dissociation through $\mathrm{TS}_{\mathrm{I}}$ to produce two coadsorbed $\mathrm{H}$ species on neighboring top-M and top- $\mathrm{C}$ sites, finally followed by the formation of the $\mathrm{H}_{2, \text { Kubas }}$ through

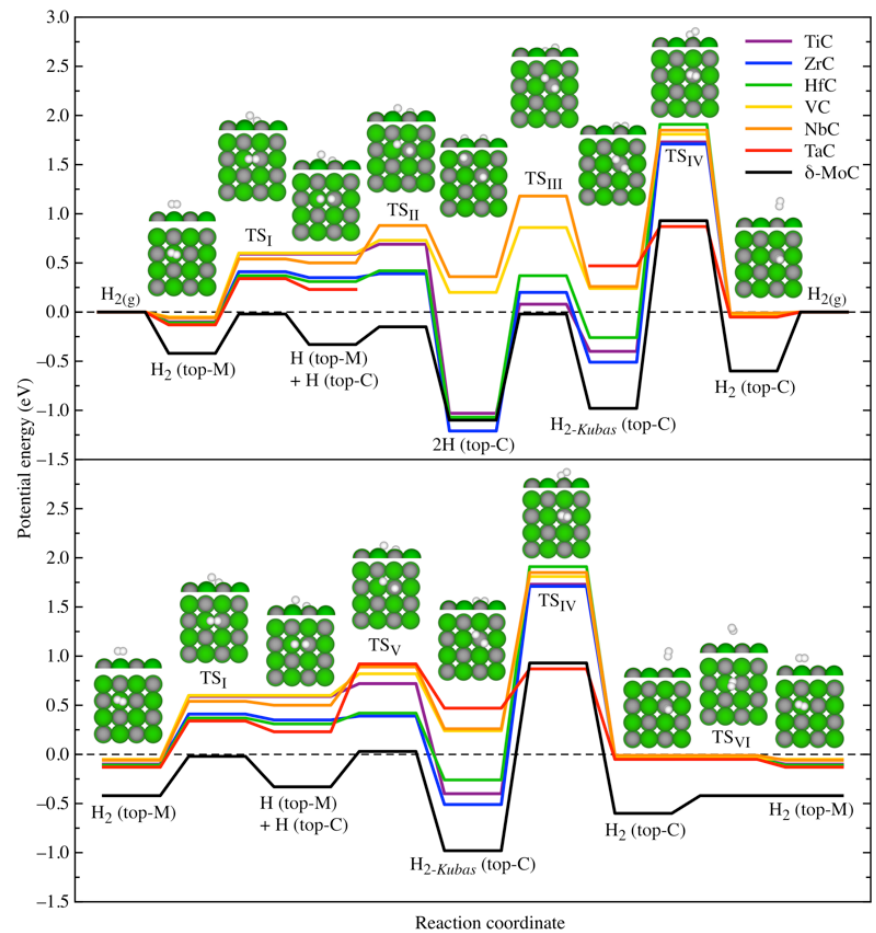

Figure 2 Energy profiles (PBE-D3 values including the ZPE term) for $\mathrm{H}_{2}$ adsorption, desorption, and dissociation on the (001) surface of the studied TMCs. The top diagram includes TSs I-IV, while the bottom diagram includes TSs I and IV-VI. The optimized geometries for all adsorption minima and transition states on $\mathrm{HfC}$ are also displayed. $\mathrm{Hf}, \mathrm{C}$, and $\mathrm{H}$ atoms are shown as green, grey, and white spheres, respectively.

the $\mathrm{TS}_{\mathrm{V}}$ saddle point, with reaching $\mathrm{TS}_{\mathrm{I}}$ being the rate limiting step, with energy barriers ranging 0.39 to $0.68 \mathrm{eV}$. However, once the two coadsorbed $\mathrm{H}$ species are formed, the diffusion of $\mathrm{H}$ species adsorbed on the top- $\mathrm{M}$ site towards another neighboring top- $\mathrm{C}$ site, resulting in two $\mathrm{H}$ species adsorbed on two separate top-C sites ( $\mathrm{TS}_{\|}$in Figure 1 ), would compete with the Kubas formation. The only exception to this general behavior is $\mathrm{TaC}$. As previously stated, this carbide adsorbs $\mathrm{H}$ species more strongly on top- $\mathrm{M}$ sites than on top-C sites. Therefore, on $\mathrm{TaC}$, the most likely scenario is $\mathrm{H}_{2}$ adsorption leading to two $\mathrm{H}$ adatoms on two separate top-M sites. This configuration can be reached from a physisorbed $\mathrm{H}_{2}$ molecule on the top-M in a single step, i.e., through $\mathrm{TS}_{\mathrm{VII}}$, with an energy barrier of $0.82 \mathrm{eV}$, or in two consecutive steps, i.e., through $\mathrm{TS}_{\text {I }}$ followed by $\mathrm{TS}_{\mathrm{VIII}}$, the latter barrier being $0.42 \mathrm{eV}$, as shown in Figure 1. The optimized geometries for TSs on all studied TMC (001) surfaces are shown in Figure S2 of the ESI.

Interestingly, for all TMCs in a given group, the energy barrier for $\mathrm{H}_{2}$ dissociation through $\mathrm{TS}_{1}$ decreases when descending along a group of the periodic table. Figure 2 shows the energy profiles for the full $\mathrm{H}_{2}$ dissociation scenario except for the two processes involving $\mathrm{TS}_{\mathrm{VII}}$ and $\mathrm{TS}_{\mathrm{VIII}}$, as these are only important for $\mathrm{TaC}$, and shown in Figure S3 of the ESI. As deduced from Figure 2, clearly, the most stable configuration for all group IV TMCs and $\delta$-MoC is the coadsorption of two $\mathrm{H}$ species adsorbed on top-C, while $\mathrm{H}_{2}$ adsorbed on either top-C or top- $\mathrm{M}$ exhibit the lowest energy configuration on group $\mathrm{V}$ 
TMCs. Note also that, on all TMCs except $\mathrm{NbC}$, the $\mathrm{H}_{2, \text { Kubas }}$ configuration is higher in energy than that for the coadsorption of two $\mathrm{H}$ species both on top-C; on $\mathrm{NbC}$ the $\mathrm{H}_{2, \text { Kubas }}$ is $0.10 \mathrm{eV}$ lower. However, it is on $\delta$-MoC that $\mathrm{H}$ on top-C position and the $\mathrm{H}_{2}$ Kubas situation should be regarded as competitive.

In summary, the present work demonstrates that group $\mathrm{V}$ TMCs are probably not the best candidates for efficient hydrogenation reactions, given the very low physisorption energies of $\mathrm{H}_{2}$ and the endoergicity of $\mathrm{H}_{2, \text { Kubas }}$ formation and of the $\mathrm{H}_{2}$ dissociation. In fact, for group $\mathrm{V}$, the only dissociated state which is lower in energy than $\mathrm{H}_{2}$ in the gas-phase is the coadsorption of two $\mathrm{H}$ species on two top-M sites in TaC. On the other hand, group IV TMCs are likely to be good candidates to replace noble metals as heterogeneous catalysts for hydrogenation reactions, see comparison of energy barriers in Table S3 in ESI. Although group IV TMCs also exhibit very low $\mathrm{H}_{2}$ physisorption energies, the adsorption energies for dissociated $\mathrm{H}$ states or the $\mathrm{H}_{2, \text { Kubas }}$ configuration are much stronger. Finally, among the seven studied TMCs, $\delta$-MoC emerges as the best candidate since it exhibits moderate

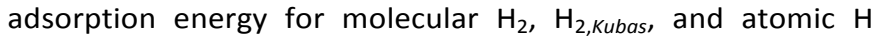
entities, plus features the lowest energy barrier for $\mathrm{H}_{2}$ dissociation on top-M site. Additionally, our results show that on the group IV TMCs and $\delta$-MoC predicted as good candidates for hydrogenation reactions, the coadsorption of two $\mathrm{H}$ species on two separate top-C sites is always preferred compared to the formation of $\mathrm{H}_{2, \text { Kubas. }}$. Moreover, the direct formation of $\mathrm{H}_{2, \text { Kubas }}$ modes from physisorbed $\mathrm{H}_{2}$ on top-C is energetically hindered. To summarize, present results show that, for hydrogenation reactions, TMCs can outperform late transition metals featuring comparable or even smaller energy barriers for $\mathrm{H}_{2}$ dissociation.

\section{Conflicts of interest}

There are no conflicts to declare.

\section{Notes and references}

1 I. Kojima, E. Miyazaki, Y. Inoue and I. Yasumori, J. Catal., 1979, 59, 472-474.

2 G. Fan, X. Li, C. Xu, W. Jiang, Y. Zhang, D. Gao, J. Bi and Y. Wang, Nanomaterials, 2018, 8, 141-154.

3 A. B. Vidal, L. Feria, J. Evans, Y. Takahashi, P. Liu, K. Nakamura, F. Illas and J. A. Rodriguez, J. Phys. Chem. Lett., 2012, 3, 2275-2280.

4 J. A. Rodriguez, J. Evans, L. Feria, A. B. Vidal, P. Liu, K. Nakamura and F. Illas, J. Catal., 2013, 307, 162-169.

5 H. Prats, R. A. Gutierrez, J. J. Piñero, F. Viñes, S. T. Bromley, P. J. Ramirez, J. A. Rodriguez and F. Illas, J. Am. Chem. Soc. 2019, 141, 5303-5313.

6 M. G. Quesne, A. Roldán, N. H. de Leeuw and C. R. A. Catlow, Phys. Chem. Chem. Phys., 2018, 20, 6905-6916.

7 J. Spielmann, F. Buch and S. Harder, Angew. Chem. Int. Ed., 2008, 120, 9576-9580.

8 S. T. Oyama, Catal. Today, 1992, 15, 179-200.
9 S. Posada-Pérez, R. A. Gutiérrez, Z. Zuo, P. J. Ramírez, F. Viñes, P. Liu, F. Illas and J. A. Rodríguez, Catal. Sci. Technol., 2017, 7, 5332-5342.

10 J. A. Rodríguez, P. Liu, D. J. Stacchiola, S. D. Senanayake, M. G. White and J. G. Chen, ACS Catal., 2015, 5, 6696-6706.

11 K. Aydin and R. Kenanoğlu, Int. J. Hydrog. Energy, 2018, 26, 14047-14058.

12 M. J. Burk, Y. M. Wang and J. R. Lee, J. Am. Chem. Soc., 2018, 21, 5142-5143.

13 F. Costanzo, P. L. Silvestrelli and F. Ancilotto, J. Chem. Theory Comput., 2012, 8, 1288-1294.

14 E. Florez, T. Gomez, P. Liu, J. A. Rodriguez, F. Illas, ChemCatChem, 2010, 2, 1219-1222.

15 C. V. J. Skipper, A. Hamaed, D. M. B Antonelli and N. Kaltsoyannis, Dalton Trans., 2012, 41, 8515-8523.

16 S. K. Estreicher, J. L. McAfee, P. A. Fedders, J. M. Pruneda and P. Ordejón, Physica B, 2001, 308, 202-205.

17 G.-Q. Yu, B.-Y. Huang, X. Chen, D. Wang, F. Zheng and X.-B. Li, J. Phys. Chem. C, 2019, 123, 21878-21887.

18 J. P. Perdew, K. Burke and M. Ernzerhof, Phys. Rev. Lett., 1996, 77, 3865-3868.

19 S. Grimme, J. Antony, S. Ehrlich and H. Krieg, J. Chem. Phys., 2010, 132, 154104.

20 G. Kresse and J. Furthmüller, Phys. Rev. B., 1996, 54, 1116911186.

21 H. Prats, S. Posada-Pérez, J. A. Rodriguez, R. Sayós and F. Illas, ACS Catal., 2019, 9, 9117-9126.

22 G. Henkelman, B. P. Uberuaga and H. Jónsson, J. Chem. Phys., 2000, 113, 9901-9904.

23 A. H. Larsen, J. J. Mortensen, J. Blomqvist, I. E. Castelli, R. Christensen, M. Dułak, J. Friis, M. N. Groves, B. Hammer, C. Hargus, E. D. Hermes, P. C. Jennings, P. B. Jensen, J. Kermode, J. R. Kitchin, E. L. Kolsbjerg, J. Kubal, K. Kaasbjerg, S. Lysgaard, J. B. Maronsson, T. Maxson, T. Olsen, L. Pastewka, A. Peterson, C. Rostgaard, J. Schiøtz, O. Schütt, M. Strange, K. S. Thygesen, T. Vegge, L. Vilhelmsen, M. Walter, Z. Zeng and K. W. Jacobsen, J. Phys. Condens. Matter, 2017, 29, 273002

24 S. Smidstrup, A. Pedersen, K. Stokbro and H. Jónsson, J. Chem. Phys., 2014, 140, 214106.

25 A. A. Koverga, E. Flórez, L. Dorkis and J. A. Rodriguez, J. Phys. Chem. C, 2019, 123, 8871-8883.

26 F. Silveri, M. G. Quesne, A. Roldán, N. H. de Leeuw and C. R. A. Catlow, Phys. Chem. Chem. Phys., 2019, 21, 5335-5343.

27 J. J. Piñero, P. J. Ramírez, S. T. Bromley, F. Illas, F. Viñes and J. A. Rodriguez, J. Phys. Chem. C, 2018, 122, 28013-28020.

28 F. Viñes, C. Sousa, P. Liu, J. A. Rodriguez and F. Illas, J. Chem. Phys., 2005, 122, 174709.

29 B. Hammer, Phys. Rev. B 2011, 63, 205423.

30 A. Gro $\beta$, J. Chem. Phys. 2011, 135, 174707. 